\title{
Implanted Occipital Nerve Stimulators
}

Joseph Jasper, MD ${ }^{1}$ and Salim Hayek MD, $\mathrm{PhD}^{2}$

From: ${ }^{1}$ Advanced Pain Medicine Physicians, Tacoma, WA; ${ }^{2}$ Division

of Pain Medicine, Department of Anesthesiology, University Hospitals of Cleveland, Case Western Reserve University; and Outcomes Research Consortium

Dr. Jasper is Medical Director, Advanced Pain Medicine Physicians, Tacoma, WA.

Dr. Hayek is with the Division of

Pain Medicine, Department of Anesthesiology, University Hospitals of Cleveland, Cleveland, $\mathrm{OH}$; and a member of the Outcomes Research

Consortium, Cleveland, $\mathrm{OH}$.

Address correspondence: Joseph F. Jasper, MD 1628 South Mildred St., \#105 Tacoma, WA 98465-1613

Email: apmedicine@qwest.net

Disclaimer: There was no external funding in the preparation of this manuscript.

Conflict of interest: None.

Manuscript received: 11/06/2007

Revised manuscript received: $12 / 03 / 2007$

Accepted for publication:01/04/2008

Free full manuscript: www.painphysicianjournal.com
Background: Chronic headache represents a very significant public health and economic issue. One treatment modality for chronic refractory headache involves the use of subcutaneous implanted neurostimulator leads in the occipital region. Varied types of headache etiologies including migraine, transformed migraine, chronic daily headache, cluster headache, hemicrania continua, occipital neuralgia, and cervicogenic headache have been studied with peripheral nerve field stimulation and found responsive to stimulation of the suboccipital region, known commonly as occipital nerve stimulation (ONS).

Objective: To evaluate the strength of evidence that occipital nerve stimulation is an effective treatment of benign headache.

Study Design: A systematic review of occipital nerve stimulation for the treatment of chronic headache.

Methods: A systematic review of the literature through EMBASE and PubMed/Medline for clinical studies was performed. The Agency for Healthcare Research and Quality (AHRQ) criteria was utilized to assess the evidence regarding occipital nerve stimulators and arrives at conclusions as to their efficacy in the treatment of headache. Studies were also graded using AHRQ criteria. The overall body of evidence was then graded on a 5 level scale from Level I (conclusive), Level II (strong), Level III (moderate), Level IV (limited) to Level V (indeterminate).

Results: Ten observational studies, of which 4 were prospective, and a number of case series, case reports, and reviews were identified. No randomized controlled trials (RCT) were identified. All of the articles reported positive outcomes including improved pain relief, reduced frequency, intensity, and duration of headaches with reduced medication consumption. ONS was reportedly successful for $70-100 \%$ of patients. Reduction of pain in patients with occipital headaches and transformed migraine is significant and rapid; for cluster patients the improvement may be less dramatic and it may take several months of occipital stimulation to achieve relief.

No long-term adverse events occurred. Several short-term incidents occurred including infection, lead displacement, and battery depletion. The body of evidence as a whole is a level of strength of IV, limited.

Conclusion: ONS is a useful tool in the treatment of chronic severe headaches with at least Level IV (limited) evidence based on multiple positive studies.

Key Words: headache, chronic daily headache, occipital neuralgia, cervicogenic headache, migraine, transformed migraine, cluster headache, neuromodulation, neurostimulation, stimulator, peripheral nerve stimulation.

Pain Physician 2008; 11:187-200 
H eadache is a major worldwide health problem disabling millions of people and resulting in substantial economic burdens. The yearly prevalence of headache, as measured in pooled studies from multiple countries, may be as high as $51 \%$. Migraine occurs in $14 \%$ and chronic daily headache (> 15 days per month) in $4 \%$ of adults (1). In the United States, $6 \%$ of men and $18 \%$ of women suffer from migraine headaches resulting in 3.8 bed rest days per year for men and 5.6 days for women and accounting for a total of 112 million bedridden days. The overall societal cost of migraine alone is comparable to that of diabetes and greater than that for asthma (2). According to a report by the WHO, a patient suffering from a severe migraine attack is considered to be as disabled as a patient with dementia, active psychosis, or quadriplegia (3). The yearly indirect costs of migraine to employers in the US amount to about $\$ 13$ billion in missed work days and impaired work function; the direct medical costs are about $\$ 1$ billion of which about $\$ 300$ million are spent on prescription drugs. The figures for indirect costs are underestimates given that several components of the disease burden were not captured (2). Since indirect costs (missed work and reduced productivity) account for over $90 \%$ of the total costs of migraine (2), the overall societal impact dictates identifying more effective preventive and therapeutic measures to treat chronic headache.

Peripheral nerve stimulation has been successfully used in the management of intractable headaches. Intractable headaches may be attributed to a variety of sources from tumors and intracranial bleeds to benign primary headaches. Up to $5 \%$ of primary headache patients suffer from chronic daily headaches and $1 \%$ of patients do not respond well to traditional oral medication regimens $(4,5)$. Peripheral nerve stimulation in the occipital region has been used successfully in the treatment of a number of headache entities including cervicogenic headache, occipital neuralgia, transformed migraine, hemicrania continua, and cluster headaches. This technique involves the placement of subcutaneous neuroelectrodes in the C1-C2 region of the posterior cervical spine. Convergence of afferents from cervical nerves C1-C3 with trigeminal afferents, at the level of second order neurons, is believed to result in the perception of headache consequent to activation of these nerves $(6,7)$. Migraine is no longer thought to be due to a dysfunction of blood vessels; rather it is thought to be due to a dysfunction of the brainstem and brain hypersensitivity (8). Weiner (5) es- timated that perhaps $50 \%$ of patients with intractable migraine may be treatable with neurostimulation.

Understanding the nerve supply of the various upper cervical and occipital structures is essential for appropriate management of patients with cervicogenic headache. Abolition of the headache following a diagnostic blockade of a cervical structure or its nerve supply using place or other adequate controls, is 1 of the IHS's (International Headache Society) diagnostic criteria for this entity. Though lacking a cutaneous innervation and originally thought not to be a sensory nerve, the C1 spinal nerve supplies some sensory innervation to deep somatic tissues in the suboccipital region. These include the short muscles of the occipital triangle through its dorsal ramus; sternocleidomastoid, trapezius, and atlanto-occipital joint through its ventral ramus; and median atlanto-axial joint, dura mater, and vertebral artery through the sinuvertebral nerve (in combination with sinuvertebral nerves of C2 and ( 3 ). The dorsal ramus of the C2 spinal nerve supplies the splenius capitis and semispinalis capitis and ultimately becomes the greater occipital nerve. Its ventral ramus supplies articular branches to the lateral atlanto-axial joint as well as prevertebral muscles, sternocleidomastoid, and trapezius. The dorsal ramus of C3 has 3 significant branches: the lateral, deep medial, and superficial medial. The lateral branch supplies the splenius capitis and cervicis and the longissimus capitis; its deep medial branch innervates the semispinalis cervicis and multifidus; the superficial medial branch innervates the semispinalis capitis. This latter branch is also known as the third occipital nerve and supplies the C2-C3 zygapophyseal joint and the skin over the suboccipital region (7). Convergent excitation appears to play a role in pain referral from cervical structures as stimulation of these nerves produces excitation of second order neurons in the trigeminocervical complex, resulting in headache $(9,10)$.

Neurostimulation of peripheral afferents of C1-C3, as in occipital nerve stimulation, may result in headache relief by affecting the trigeminocervical complex neurons (11). The use of implanted peripheral neurostimulators to treat headache patients is reviewed in this systematic review.

\section{Methods}

The general method of review follows that outlined by Manchikanti et al $(12,13)$, based on the criteria of the Agency for Healthcare Research and Quality (AHRQ) criteria. Table 1 illustrates criteria for evaluating randomized and observational studies. 


\section{Data Sources}

English language studies searched in MEDLINE and EMBASE 1966 - 2007 and additional references were gleaned from retrieved articles. A computerized search of the PubMed and EMBASE databases from January 1966 to October 2007 for English-language publications was conducted using the key words occipital nerve or headache separately with stimulation, peripheral nerve stimulation, occipital nerve stimulation, or occipital neurostimulation. The reference lists of the retrieved articles were reviewed for additional studies, as were review articles on the subject. The earliest human application of spinal cord stimulation occurred in 1967 (14) and the earliest report on peripheral nerve stimulation in 1977 (15) justifying the timeline of reference search.

\section{Study Selection}

All articles were triaged for inclusion by either author for suitability prior to review. Studies were selected for inclusion in this synthesis if the methods section clearly indicated that peripheral nerve stimulation was applied as the primary treatment modality for headache.

\section{Selection Criteria} Inclusion Criteria

Randomized controlled trials and observational studies were included in the analysis of strength of evidence. Types of participants included adult patients with frequent and intense headaches of $>6$ months duration who had not adequately responded to conventional headache therapies such as injections and/or ablative techniques to the structures involved in the generation and maintenance of headache, medications, and psychological interventions.

Exclusion Criteria

Narrative reports lacking study data, case reports, and technical reports with follow-up of less than 3 months were excluded from data analysis.

\section{Outcome Measures}

The primary outcome measure sought was measurement of pain relief; secondary outcome measures were of functional nature. The following functional measures were considered acceptable for inclusion: headache frequency, intensity, and duration; medication use; Morphine Dose Equivalents (MDE); number of doctor or ER visits; ratio of trial to permanent implantation; complications; the Neck Pain Disability Ques- tionnaire (NPDQ); the Migraine Disability Assessment (MIDAS) scores; return to work; and Quality of Life. Short-term relief was considered less than 6 months. Intermediate-term relief was regarded as 6 months to 2 years. Long-term relief was considered in terms of longer than 2 years outcomes (16-18).

\section{Method of Review}

The authors independently scored each article using the method described by AHRQ and Manchikanti et al $(12,13)$. Any differences in rating between the reviewers were reconciled with a third party not involved in the assessment.

Methodological Quality

Criteria used in reviewing and grading the literature are shown in Table 1. Articles were classified as randomized controlled trials (RCT), observational prospective, observational retrospective, or other, including both narratives and technical reports. Only RCT and observational studies were rated. The appropriate rating forms were used to grade RCTs and observational studies. Fractional scores were converted to percentage for overall grading of the prospective and retrospective literature.

\section{Overall Strength of Evidence}

The strength of evidence in the reviewed articles was assessed as described elsewhere and illustrated in

Table 1. Domains in the Agency for Healthcare Research and Quality (AHRQ) criteria for evaluating the quality of individual studies.

\begin{tabular}{|l|l|}
\hline \multicolumn{1}{|c|}{$\begin{array}{c}\text { Randomized Controlled } \\
\text { Studies }\end{array}$} & \multicolumn{1}{|c|}{ Observational Studies } \\
\hline Study question & Study question \\
\hline Study population & Study population \\
\hline Randomization & Comparability of subjects \\
\hline Blinding & Exposure or intervention \\
\hline Interventions & Outcome measures \\
\hline Outcomes & Statistical analysis \\
\hline Statistical analysis & Results \\
\hline Results & Discussion \\
\hline Discussion & Funding or sponsorship \\
\hline Funding or sponsorship & \\
\hline
\end{tabular}

Italics indicate elements of critical importance in evaluating grading systems according to empirical validation research or standard epidemiological methods.

Reproduced from West et al (12) 
Table 2. Criteria for rating the overall strength of body of evidence.

\begin{tabular}{|l|l||}
\hline \hline Domain & Definition \\
\hline Quality & $\begin{array}{l}\text { The quality of all relevant studies for a given topic, where "quality" is defined as the extent to which a study's design, } \\
\text { conduct, and analysis has minimized selection, measurement, and confounding biases }\end{array}$ \\
\hline Quantity & $\begin{array}{l}\text { The magnitude of treatment effect } \\
\text { The number of studies that have evaluated the given topic } \\
\text { The overall sample size across all included studies }\end{array}$ \\
\hline Consistency & For any given topic, the extent to which similar findings are reported from work using similar and different study designs \\
\hline
\end{tabular}

Reproduced from Manchikanti et al (13) with permission

Table 3. The National Health and Medical Research Council (NHMRC) keypoints in consideration of level of evidence.

- Resolution of differences in the conclusions reached about effectiveness from studies at differing levels of evidence or within a given level of evidence.

- Resolution of the discrepancies is an important task in the compilation of an evidence summary.

- Inclusion of biostatistical and epidemiological advice on how to search for possible explanation for the disagreements before data are rejected as being an unsuitable basis on which to make recommendations.

- Recognition of the fact that it may not be feasible to undertake randomized controlled trials in all situations.

- Guidelines should be used on the best available evidence.

- Recognition of the fact that it may be necessary to use evidence from different study designs for different aspects of the treatment.

Reproduced from Manchikanti et al (13) with permission
Table $2(12,13)$. Factors considered in establishing the level of evidence and the rating system used in determining the level of evidence are illustrated in Tables 3 and 4 respectively.

\section{Results}

\section{Literature Search}

A total of 813 articles were obtained in the search; upon review by the authors there were 13 relevant articles identified. There were another 10 reports that were retrieved from cross references.

\section{Methodological Review Findings}

The evaluation process according to review guidelines yielded a total of 4 observational prospective case series (1 cluster), 8 retrospective case series (2 cluster), 3 case reports, 3 narrative reports, and 3 technical reports. The articles on the use of implanted neurostimulators for headache are reviewed in Tables $5-7$. No RCTs were identified and only observational studies were thus rated. Prospective and retrospective reports on headache entities other than cluster are depicted in Table 5. Studies on patients with cluster headaches are

Table 4. Levels of evidence.

\begin{tabular}{|l|l||}
\hline \hline Level I & $\begin{array}{l}\text { Conclusive: Research-based evidence with multiple relevant and high-quality scientific studies or consistent reviews of } \\
\text { meta-analyses. }\end{array}$ \\
\hline Level II & $\begin{array}{l}\text { Strong: Research-based evidence from at least 1 properly designed randomized, controlled trial; or research-based } \\
\text { evidence from multiple properly designed studies of smaller size; or multiple low quality trials. }\end{array}$ \\
\hline Level III & $\begin{array}{l}\text { Moderate: a) Evidence obtained from well-designed pseudo-randomized controlled trials (alternate allocation or some } \\
\text { other method); b) evidence obtained from comparative studies with concurrent controls and allocation not randomized } \\
\text { (cohort studies, case-controlled studies, or interrupted time series with a control group); c) evidence obtained from } \\
\text { comparative studies with historical control, 2 or more single-arm studies, or interrupted time series without a parallel } \\
\text { control group. }\end{array}$ \\
\hline Level IV & $\begin{array}{l}\text { Limited: Evidence from well-designed non-experimental studies from more than 1 center or research group; or } \\
\text { conflicting evidence with inconsistent findings in multiple trials. }\end{array}$ \\
\hline Level V & $\begin{array}{l}\text { Indeterminate: Opinions of respected authorities, based on clinical evidence, descriptive studies, or reports of expert } \\
\text { committees. }\end{array}$ \\
\hline
\end{tabular}

Reproduced from Manchikanti et al (13) with permission 
illustrated in Table 6. Narrative and case reports worth mentioning are presented in Table 7, but these were not included in data analysis. Outcome measures varied from one study to the next. Some made use only of reduction in average Pain VAS, while others were more sophisticated assessing headache frequency and intensity, NPDQ, MIDAS, MDE, and other medication use reports or work status. Most mentioned complications when they occurred. Complications are summarized in Table 8. Follow-up periods ranged from 3 months minimum to several years

Popeney and Alo (11) prospectively studied 25

Table 5: Summary of case series or technical reports on patients with occipital neuralgia or transformed migraine implanted with ONS. The double line separates prospective from retrospective studies.

\begin{tabular}{|c|c|c|c|c|c|c|c|c|c|c|}
\hline Study & $\begin{array}{l}\text { Study } \\
\text { Type }\end{array}$ & \begin{tabular}{|l} 
AHRQ \\
Score
\end{tabular} & $\begin{array}{l}\text { Type of } \\
\text { Headache }\end{array}$ & $\begin{array}{l}\text { Trial } \\
\text { Number }\end{array}$ & $\begin{array}{l}\text { Implant } \\
\text { Number }\end{array}$ & $\begin{array}{l}\text { Short- } \\
\text { Term } \\
\text { Relief }\end{array}$ & $\begin{array}{l}\text { Intermed- } \\
\text { Term } \\
\text { Relief }\end{array}$ & $\begin{array}{l}\text { Follow- } \\
\text { up } \\
\text { Period }\end{array}$ & $\begin{array}{l}\text { Result } \\
\text { Short- } \\
\text { Term }\end{array}$ & $\begin{array}{l}\text { Result } \\
\text { Intermed- } \\
\text { Term }\end{array}$ \\
\hline $\begin{array}{l}\text { Melvin et } \\
\text { al. (20) }\end{array}$ & P, CS & $9 / 11$ & $\begin{array}{l}\text { C2 Mediated } \\
\text { headache }\end{array}$ & 16 & 11 & $64 \%$ & N/A & $12 \mathrm{wk}$ & $\mathrm{P}$ & NA \\
\hline $\begin{array}{l}\text { Popeney } \\
\text { and Alo } \\
(11)\end{array}$ & $\begin{array}{l}\mathrm{P}, \mathrm{CS}, \\
\mathrm{pr} / \mathrm{po}\end{array}$ & $9 / 11$ & $\begin{array}{l}\text { Transformed } \\
\text { migraine }\end{array}$ & 25 & $25(100 \%)$ & $\begin{array}{l}100 \%>50 \% \\
\text { MIDAS } \\
88.7 \% \downarrow\end{array}$ & $\begin{array}{l}20>75 \% \\
5>50 \% \\
\text { MIDAS 15=I } \\
1=\mathrm{II} \\
4=\mathrm{III} \\
5=\mathrm{IV}\end{array}$ & $\begin{array}{l}9-36 \mathrm{~m} \\
\text { Avg } \\
=18.3 \mathrm{~m}\end{array}$ & $\mathrm{P}$ & $\mathrm{P}$ \\
\hline $\begin{array}{l}\text { Oh et al. } \\
(19) \\
\text { (paddle) }\end{array}$ & P, T, CS & $10 / 11$ & $\begin{array}{l}\text { Occipital } \\
\text { neuralgia }\end{array}$ & $\begin{array}{l}20 ; \\
10=\mathrm{On} \\
10=\mathrm{Tm}\end{array}$ & 20 & $\begin{array}{l}19 / 20 \text { with } \\
80-100 \% \\
\text { relief }\end{array}$ & $\begin{array}{l}18 / 20 \\
90 \% \\
2 \text { expl }\end{array}$ & $8 m-5 y$ & $\mathrm{P}$ & $\mathrm{P}$ \\
\hline $\begin{array}{l}\text { Weiner et al } \\
(34)\end{array}$ & $\begin{array}{l}\text { R, CS, } \\
\mathrm{pr} / \mathrm{po}\end{array}$ & $6 / 11$ & $\begin{array}{l}\text { Occipital } \\
\text { neuralgia }\end{array}$ & 13 & 33 & 20 & $\begin{array}{l}55 \% \text { exc } \\
27 \% \text { good } \\
15 \% \text { fair }\end{array}$ & & & $\mathrm{P}$ \\
\hline $\begin{array}{l}\text { Weiner and } \\
\text { Reed (36) }\end{array}$ & $\begin{array}{l}\text { R, CS, } \\
\mathrm{pr} / \mathrm{po}\end{array}$ & $8 / 11$ & $\begin{array}{l}\text { Occipital } \\
\text { neuralgia }\end{array}$ & 6 & 13 & $\begin{array}{l}100 \% \\
\text { Good- } \\
\text { excellent }\end{array}$ & $\begin{array}{l}100 \% \\
\text { Good- } \\
\text { excellent }\end{array}$ & $6 m-17 y$ & $\mathrm{P}$ & $\mathrm{P}$ \\
\hline $\begin{array}{l}\text { Matharu et } \\
\text { al. (21) }\end{array}$ & $\mathrm{R}, \mathrm{CS}$ & $8 / 10$ & Migraine & 8 & $8 / 8$ & $\begin{array}{l}100 \% \\
\text { Good- } \\
\text { Excellent }\end{array}$ & $\begin{array}{l}100 \% \\
\text { Good- } \\
\text { excellent }\end{array}$ & $1-4 y$ & $\mathrm{P}$ & $\mathrm{P}$ \\
\hline $\begin{array}{l}\text { Kapural et } \\
\text { al. (22) } \\
\text { (paddle) }\end{array}$ & R, T, CS & $8 / 11$ & $\begin{array}{l}\text { Cervicogenic } \\
\text { headache }\end{array}$ & 4 & 6 & $\begin{array}{l}100 \% \\
\text { VAS } \downarrow 8.6 \text { to } \\
2.5 \\
\text { PDI } \downarrow 49 \\
\text { to } 14\end{array}$ & N/A & $3 \mathrm{mos}$ & $\mathrm{P}$ & N/A \\
\hline $\begin{array}{l}\text { Rodrigo- } \\
\text { Royo et al. } \\
(29)\end{array}$ & $\begin{array}{l}\text { CR x 4, } \\
\mathrm{pr} / \mathrm{po}\end{array}$ & $8 / 11$ & $\begin{array}{l}\text { Occipital } \\
\text { neuralgia }\end{array}$ & 14 & 4 & $\begin{array}{l}100 \% \\
4 / 4\end{array}$ & $100 \% 3 / 3$ & $\begin{array}{l}2,6 \\
9,16 \\
\text { months }\end{array}$ & & \\
\hline $\begin{array}{l}\text { Slavin et al. } \\
(30)\end{array}$ & $\begin{array}{l}\text { R, CS, } \\
\mathrm{pr} / \mathrm{po}\end{array}$ & $7 / 11$ & $\begin{array}{l}\text { Occipital } \\
\text { neuralgia }\end{array}$ & $\begin{array}{l}8 \\
\text { Perc lead }\end{array}$ & 10 & $10 / 10>50 \%$ & $\begin{array}{l}8 / 10>50 \% \\
210 \text { compi }\end{array}$ & $5-32 \mathrm{~m}$ & $\mathrm{P}$ & $\mathrm{P}$ \\
\hline $\begin{array}{l}\text { Johnstone } \\
\text { and Sundaraj } \\
\text { (32) }\end{array}$ & $\mathrm{R}, \mathrm{CS}$ & $7 / 11$ & $\begin{array}{l}\text { Occipital } \\
\text { neuralgia }\end{array}$ & 13 & $\begin{array}{l}7 \\
\text { paddle }\end{array}$ & $\begin{array}{l}5 / 7 \text { had } \downarrow \\
\text { VAS }\end{array}$ & $\begin{array}{l}2 / 7 \text { returned } \\
\text { to work }\end{array}$ & 6-47 mo & & $\begin{array}{l}\mathrm{P} \\
2 / 7 \text { infection }\end{array}$ \\
\hline
\end{tabular}

$T=$ Technical report; $C R, C S=C a s e$ report, Case series; $N R=$ Narrative review; $R=$ Retrospective; $P=$ Prospective; $p o=$ post-test measurements only; $\mathrm{pr} / \mathrm{po}=\mathrm{pre}$ and post test measurements; $R A=$ randomized; $P C=$ Placebo controlled; $D B=$ Double blind; $C=C o n t r o l ; N / A=$ Not available; $P=P o s i t i v e ; N=N e g a t i v e ; O n=O c c i p i t a l$ neuralgia; Tm=Transformed migraine; MIDAS=Migraine Disability Assessment 
Table 6: Summary of studies on patients with cluster headache or hemicrania continua implanted with ONS. The double line separates prospective from retrospective studies.

\begin{tabular}{|l|l|l|l|l|l|l|l|l|l||}
\hline Study & $\begin{array}{l}\text { Study } \\
\text { Type }\end{array}$ & $\begin{array}{l}\text { AHRQ } \\
\text { Score }\end{array}$ & $\begin{array}{l}\text { Type of } \\
\text { Headache }\end{array}$ & $\begin{array}{l}\text { Implant } \\
\text { Number }\end{array}$ & $\begin{array}{l}\text { Short- } \\
\text { Term } \\
\text { Relief }\end{array}$ & $\begin{array}{l}\text { Intermed- } \\
\text { Term } \\
\text { Relief }\end{array}$ & $\begin{array}{l}\text { Follow- } \\
\text { up } \\
\text { Period }\end{array}$ & $\begin{array}{l}\text { Result } \\
\text { Short- } \\
\text { Term }\end{array}$ & $\begin{array}{l}\text { Result } \\
\text { Intermed- or } \\
\text { Long- } \\
\text { Term }\end{array}$ \\
\hline \hline $\begin{array}{l}\text { Magis et al. } \\
(26)\end{array}$ & P, CS & $9 / 11$ & Cluster & 8 & $\begin{array}{l}7 / 8 \\
2 \text { had } \\
\text { total } \\
\text { relief }\end{array}$ & $\begin{array}{l}\text { Peak effect at } 2 \\
\text { month post- } \\
\text { implant }\end{array}$ & $3-22$ mo & $\begin{array}{l}\text { P } \\
7 / 8\end{array}$ & $\begin{array}{l}\text { P } \\
6 / 8 \text { long-term } \\
\text { follow-up }\end{array}$ \\
\hline $\begin{array}{l}\text { Schwedt et } \\
\text { al. (27) }\end{array}$ & $\begin{array}{l}\text { R, CS, } \\
\text { pr/po }\end{array}$ & $9 / 10$ & $\begin{array}{l}3 \text { Cluster } \\
2 \text { Hemicrania } \\
4 \text { Continua } \\
8 \text { Migraine } \\
\text { 2 Post-Traumatic }\end{array}$ & 15 & 15 & $\begin{array}{l}\text { Significant } \downarrow \\
\text { in frequency, } \\
\text { severity, HIT and } \\
\text { MIDAS scores }\end{array}$ & 3 y & P & $\begin{array}{l}\text { P } \\
60 \% \text { lead } \\
\text { revision }\end{array}$ \\
\hline $\begin{array}{l}\text { Burns et al. } \\
\text { (23) }\end{array}$ & R, CS & $8 / 11$ & Cluster & 8 & $\begin{array}{l}\text { None } \\
\text { before } \\
\text { weeks }\end{array}$ & $\begin{array}{l}\text { 6/8 had } \\
\text { significant } \\
\text { improvement }\end{array}$ & $8-27$ mo & $\begin{array}{l}\text { P } \\
6 / 8\end{array}$ & $\begin{array}{l}\text { P; } \downarrow \text { in } \\
\text { frequency or } \\
\text { severity, not } \\
\text { duration }\end{array}$ \\
\hline
\end{tabular}

$C R, C S=C a s e$ report, Case series; $R=$ Retrospective; $P=$ Prospective; $p o=$ Post-test measurements only; $p r / p o=$ pre and post test measurements; $N / A=N o t$ available; $\mathrm{P}=$ positive.

Table 7: Summary of noteworthy narrative, technical, and case reports on patients with occipital headache or hemicrania continua implanted with ONS*.

\begin{tabular}{|c|c|c|c|c|c|c|c|c|c|c|}
\hline Study & Characteristics & $\begin{array}{l}\text { AHRQ } \\
\text { Score }\end{array}$ & $\begin{array}{l}\text { Type of } \\
\text { Headache }\end{array}$ & $\begin{array}{l}\text { Trial } \\
\text { Number }\end{array}$ & $\begin{array}{l}\text { Implant } \\
\text { Number }\end{array}$ & $\begin{array}{l}\text { Short- } \\
\text { Term } \\
\text { Relief }\end{array}$ & $\begin{array}{l}\text { Intermed- } \\
\text { Term } \\
\text { Relief }\end{array}$ & $\begin{array}{l}\text { Intermed- } \\
\text { Term } \\
\text { Period }\end{array}$ & $\begin{array}{l}\text { Result } \\
\text { Short- } \\
\text { Term }\end{array}$ & $\begin{array}{l}\text { Result } \\
\text { Intermed- } \\
\text { Term }\end{array}$ \\
\hline $\begin{array}{l}\text { Picaza et } \\
\text { al (15) }\end{array}$ & NR & NA & $\begin{array}{l}\text { Occipital } \\
\text { Neuralgia }\end{array}$ & 6 & & & $\begin{array}{l}3 / 6 \text { good- } \\
\text { excellent }\end{array}$ & & & \\
\hline $\begin{array}{l}\text { Waisbrod } \\
\text { et al (33) }\end{array}$ & $\begin{array}{l}\text { NR } \\
\text { heterogeneous }\end{array}$ & NA & $\begin{array}{l}\text { Occipital } \\
\text { Neuralgia }\end{array}$ & 1 & 1 & NA & NA & NA & $\mathrm{P}$ & NA \\
\hline $\begin{array}{l}\text { Schwedt } \\
\text { et al (25) }\end{array}$ & $\mathrm{R}, \mathrm{CR}$ & NA & $\begin{array}{l}\text { Cluster } \\
\text { Hemicrania } \\
\text { Continua }\end{array}$ & 2 & 2 & $\begin{array}{l}1 \text { had } \\
70 \% \\
\text { relief }\end{array}$ & NA & NA & $\mathrm{P}$ & NA \\
\hline $\begin{array}{l}\text { Hammer } \\
\text { and } \\
\text { Doleys } \\
(28)\end{array}$ & $\mathrm{CR}$ & NA & $\begin{array}{l}\text { Occipital } \\
\text { Neuralgia }\end{array}$ & 1 & 1 & $\begin{array}{l}1 / 1 \\
\text { excellent }\end{array}$ & $\begin{array}{l}1 / 1 \\
\text { excellent }\end{array}$ & $6 \mathrm{~m}$ & & \\
\hline $\begin{array}{l}\text { Weiner } \\
\text { (5) }\end{array}$ & $\begin{array}{l}\text { NR, R, no } \\
\text { demogr or detail }\end{array}$ & $\mathrm{NA}$ & $\begin{array}{l}\text { Review of } \\
\text { all data }\end{array}$ & & 150 & & $\begin{array}{l}70-75 \% \text { pts } \\
>50 \% \text { relief }\end{array}$ & $\begin{array}{l}\text { Up to } 12 \\
\text { years }\end{array}$ & & $\begin{array}{l}\mathrm{P} \\
\text { Migration } \\
\text { in 15\% }\end{array}$ \\
\hline
\end{tabular}

$\mathrm{CR}, \mathrm{CS}=$ Case report, Case Series; NR Narrative Review; $\mathrm{R}=$ Retrospective; $\mathrm{P}=$ Prospective; $\mathrm{po}=$ post-test measurements only; $\mathrm{pr} / \mathrm{po}=$ pre and post test measurements; $\mathrm{N} / \mathrm{A}=$ not available; $\mathrm{P}=$ positive.

*not included in rating of evidence

patients with transformed migraine. Pre- and post-implant measurements of Migraine Disability Assessment (MIDAS) scores were collected. Reportedly $100 \%$ of the patients graduated from trial to permanent implantation with cylindrical percutaneous type leads. Following surgery, $100 \%$ of implanted patients had relief of $>$ $50 \%$ and $80 \%$ of patients had relief $>70 \%$. All patients reported that their headaches were well controlled with neurostimulation and the average improvement in MIDAS scores was $88.7 \%$. A number of patients lost stimulation due to percutaneous lead migration and all those had recurrence of headache (11). This represented in effect a control group. Once their leads were replaced, all achieved good to excellent relief again. The leads used in this study were percutaneous cylindrical leads and there was a 9/25 incidence of lead migration 
Table 8. Summary of complications in patients implanted with ONS.

\begin{tabular}{|c|c|c|c|c|c|c|c|c|}
\hline & $\begin{array}{c}\text { Failed } \\
\text { trial }\end{array}$ & $\begin{array}{c}\text { Implanted } \\
\text { (F/M) }\end{array}$ & $\begin{array}{c}\text { Lead } \\
\text { migration }\end{array}$ & $\begin{array}{l}\text { Lead } \\
\text { type }\end{array}$ & $\begin{array}{l}\text { Lead fx or } \\
\text { disconnect }\end{array}$ & Infection & $\begin{array}{c}\text { Requested } \\
\text { removal }\end{array}$ & "Allergy" \\
\hline Melvin et al.(20) & $3 / 14$ & $11(9 / 2)$ & $1 / 11$ & $\mathrm{C}$ & 1 & 0 & & \\
\hline Popeney \& Aló (11) & $0 / 25$ & $25(22 / 3)$ & $9 / 25$ & $\mathrm{C}$ & $0 / 25$ & $1 / 25$ & $0 / 25$ & $0 / 25$ \\
\hline Weiner \& Reed (36) & NA & $13(8 / 5)$ & $1 / 13$ & $\mathrm{C}$ & $1 / 13$ & $1 / 13$ & $0 / 13$ & \\
\hline Slavin et al. (30) & $4 / 14$ & $10(7 / 3)$ & $1(\mathrm{x} 2) / 14$ & $\mathrm{C}$ & $0 / 10$ & $1 / 10$ & $1 / 10$ & $0 / 10$ \\
\hline Schwedt et al. (27) & $0 / 15$ & $15(12 / 3)$ & $15 / 15$ & $\mathrm{C}$ & $0 / 15$ & $0 / 15$ & $0 / 15$ & $0 / 15$ \\
\hline Burns et al. (23) & NA & $8(1 / 7)$ & $1(3 \mathrm{x}) / 8$ & $\mathrm{C}$ & $1 / 8$ & $0 / 8$ & $0 / 8$ & $0 / 8$ \\
\hline Magis et al. (26) & NA & $8(1 / 7)$ & $2 / 8$ & $\mathrm{P}$ & $0 / 8$ & $0 / 8$ & $1 / 8$ & $0 / 8$ \\
\hline $\begin{array}{l}\text { Johnstone and } \\
\text { Sundaraj. (32) }\end{array}$ & $1 / 8$ & $7(3 / 4)$ & $0 / 7$ & $\mathrm{P}$ & $0 / 7$ & $2 / 7$ & $0 / 7$ & $0 / 7$ \\
\hline Oh et al. (19) & NA & 20 & $0 / 20$ & $\mathrm{P}$ & $0 / 20$ & $2 / 20$ & $1 / 20$ & $1 / 20$ \\
\hline Kapural et al. (22) & NA & $6(2 / 4)$ & NR & $\mathrm{P}$ & NA & NA & NA & NA \\
\hline
\end{tabular}

$C=$ Cylindrical percutaneous lead; $P=$ Paddle type surgical lead; $N A=$ Not available.

and 1/25 incidence of infection. A similar self-as-control pattern during complications was repeatedly found in other studies. Patients with migrated leads in this study were implanted using paddle type leads in the study by Oh et al (19). In this study, 2 preoperative diagnostic groups were identified and treated at separate facilities, occipital neuralgia and transformed migraine. All failed to respond to medications, physical therapy, and had short-term but no long-term improvement with occipital local anesthetic field block. The occipital neuralgia group $(\mathrm{N}=10)$ was treated with unilateral paddle lead electrodes with $100 \%$ response and no lead migration. In the transformed migraine group 7/10 had previous success with percutaneously implanted cylindrical leads that migrated and all 10 were then implanted with a paddle style lead with $100 \%$ positive response. Complications occurred in 4/20. Two infections occurred, 1 treated with intravenous antibiotics and 1 with lead explantation and subsequent re-implantation; both did well long-term. Two systems were explanted by request: in 1 patient for increased overall pain despite less overall occipital pain during stimulation; the other patient developed pulse generator pocket pain, thought to have occurred secondary to metal allergy (19).

In a prospective pilot study, Melvin et al (20) implanted 11/16 trialed patients with C2-mediated occipital headaches finding not just improvement in pain, but $91 \%$ of implanted patients reported medication reduction and substantial improvements in function such as work and recreational activities. Satisfaction was also universal with no patients claiming dissatisfaction: $73 \%$ reported good to excellent results and
$27 \%$ reported fair results (20).

Matharu et al (21) conducted a semi-blinded randomized prospective controlled study on 8 migraine patients with already implanted peripheral nerve stimulators and, using pain reduction as the criterion, successful outcome. However, the primary focus of the paper was to study physiologic effects of stimulation using H215O PET scan. The study's patient group had been selected based on successful outcome after implantation of ONS for 1-4 years. Hence, the study could not be considered prospective as far as the effectiveness of ONS on pain relief. Pain level was measured as well as the time to change in pain level. The variable introduced was 1 of 3 states of stimulation: no stimulation, effective stimulation, and partial but ineffective stimulation. As patients were not told what state was being programmed but could inevitably perceive stimulation paresthesias, the study cannot be considered fully blinded. Every patient was subjected randomly to each of the 3 states 4 times for a total of 12 sessions per patient or a total of 96 sessions for the group with coordinated PET scanning, and recording of pain reports. The PET scans demonstrated definite and far reaching metabolic effects of pain and of stimulation that are distinct throughout the brain and brainstem. Further increase in stimulation consistently provided onset of a 7 point drop in VAS 10 within an average of 15 minutes. Switching off the stimulator consistently resulted in recurrence of headache pain with a rise of 7 points on a VAS pain scale of 0 to 10 (Fig. 1). The intent of the study was not to examine long-term outcomes from trial to permanent implant. All 8 patients 
(A) Sub-occipital stimulator switched off

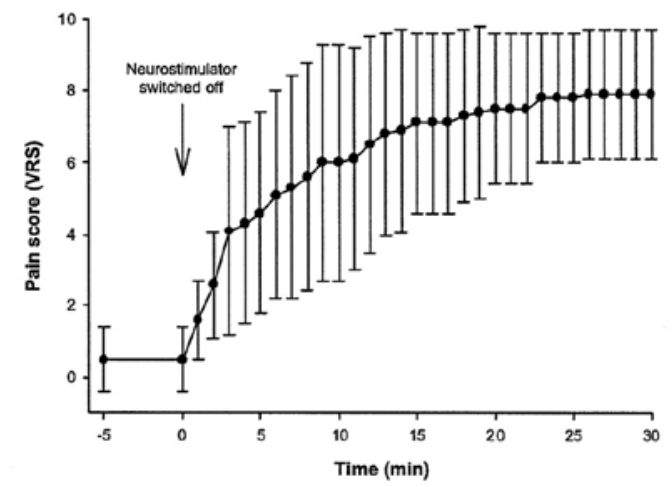

(B) Suboccipital stimulator switched on

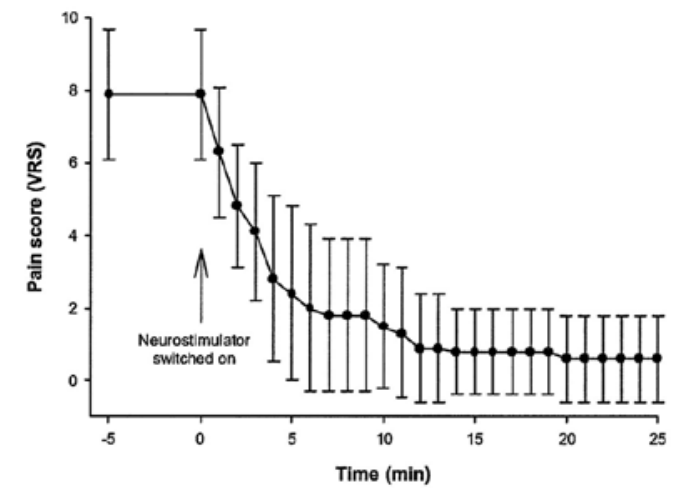

Fig. 1. Temporal headache perception response of patients implanted with suboccipital peripheral nerve stimulators to turning stimulation on or off. Reproduced with permission of Goadsby \& Oxford University Press (16). in this study were reporting long-term relief at an average follow-up from surgery of 1.5 years, though this was irrelevant to the study design. The results support a definite correlation between stimulation intensity, pain intensity, and PET findings (Figs. 2,3). There was a definite direct relationship between stimulation and pain relief and between stimulation and the appearance of PET findings. There was a definite indirect correlation between pain intensity and appearance of PET findings (21). Kapural et al (22) reported on 6 patients with severe occipital neuralgia treated with paddle type occipital peripheral nerve stimulation using a midline approach (Fig. 5). All patients had received multiple medications, occipital nerve blocks, and cervical facet blocks/radiofrequency ablation with short-term relief. There were significant improvements not only in the visual analog scores but also in Pain Disability Index (PDI) scores (22). The authors did not comment on complications using this approach.

Cluster headache and hemicrania contiunua have also been treated with occipitally placed peripheral nerve stimulators (23-25), again based on the demonstration that ONS causes stimulation of the trigeminal nucleus caudalis (6). Burns et al (23) and Magis et al (26) separately reported case series of 8 patients with chronic intractable cluster headache who received occipital nerve stimulators. Outcomes were statistically favorable, though less dramatic than migraine studies. It was notable that onset of relief for cluster patients seemed to improve over a period of weeks to months. Success was again measured as reduction of frequency, duration, and intensity of headaches as well as reduction in medication use. Unilateral stimulator
(A)

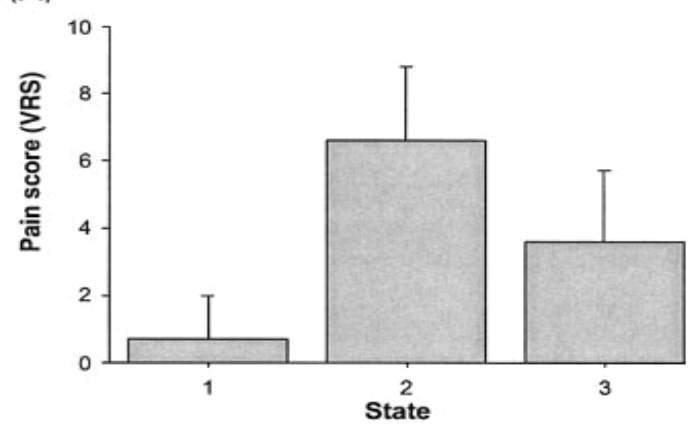

(B)

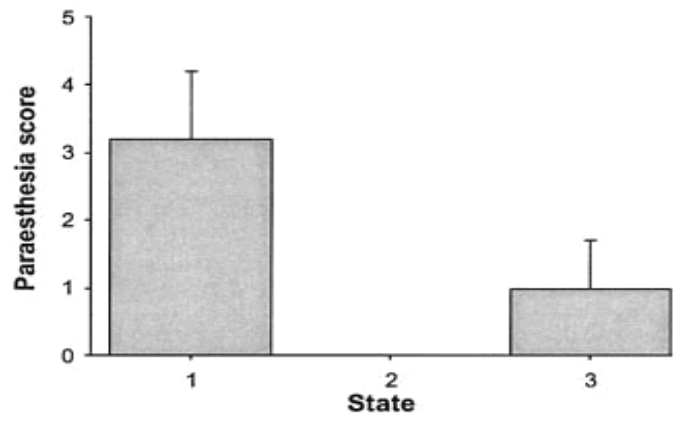

Fig. 2 . (A) mean pain scores and (B) mean scores of stimulator-induced paraesthesia by scanning states. The scanning states were (1) stimulator at optimum settings: patient pain-free or with mild pain and paraesthesia; (2) stimulator off: patient in pain and no paraesthesia; (3) stimulator partially activated: patient with intermediate levels of pain and paraesthesia. Reproduced with permission of Goadsby \& Oxford University Press (16). 


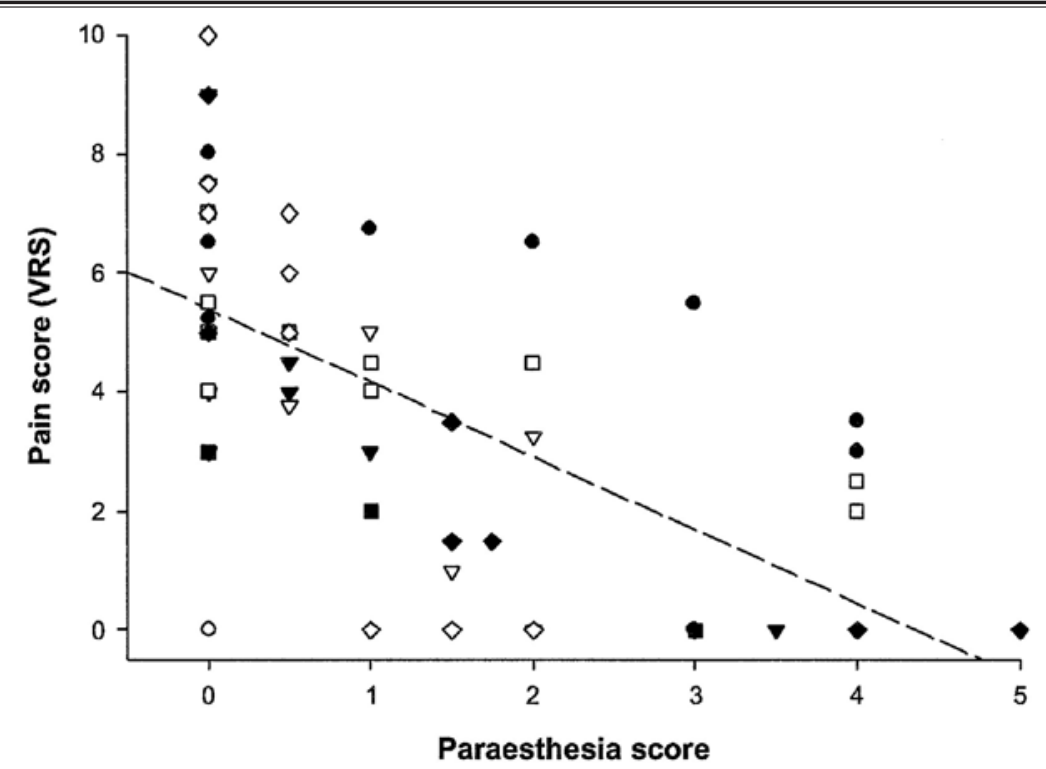

Each symbol represents the results from an individual patient. Dashed line is the plot of standard linear regression (intercept $=5.460 .3$; regression coeficient $= \pm 1.260 .2 ; \mathrm{P} \ldots-0.05 ; \mathrm{r} 2=0.38$.

Fig. 4. Graph of pain scores (VRS: $0=$ no pain; $10=$ most severe pain) versus stimulator-induced paraesthesia $(0=$ no paraesthesia, $5=$ strongest paraesthesia) in chronic migraine patients $(n=8)$. Reproduced with permission of Goadsby \& Oxford University Press (16).

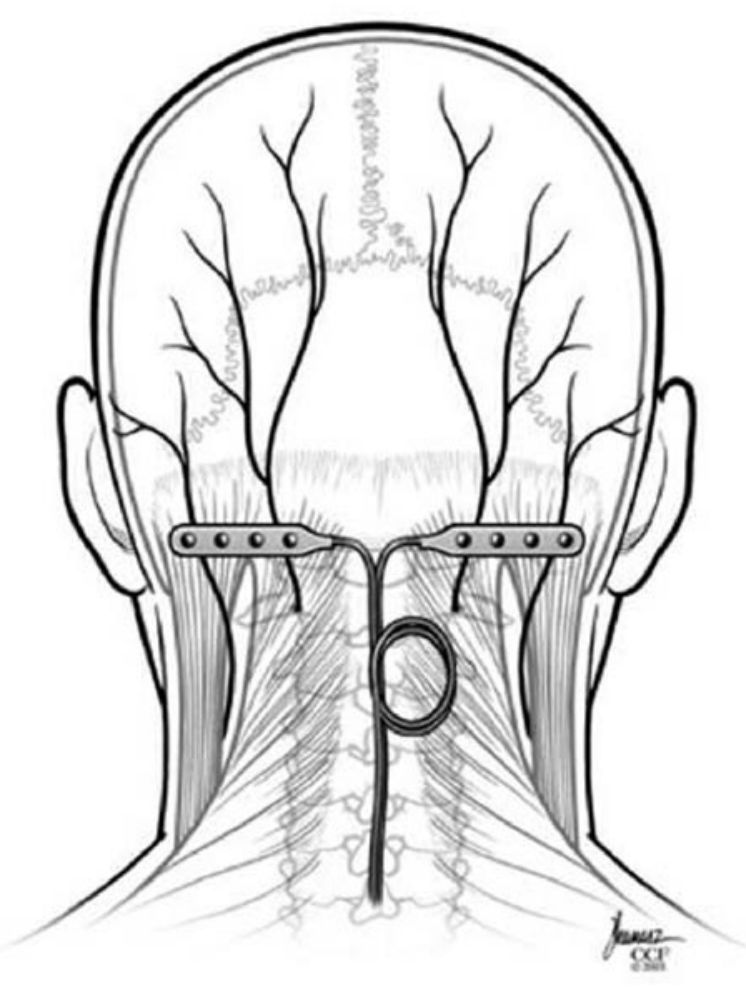

Fig 5. Illustration of placement of paddle type leads for bilateral occipital nerve stimulation. Reprinted with permission from Kapural et al (22). 
A. Dorsal rostral pons

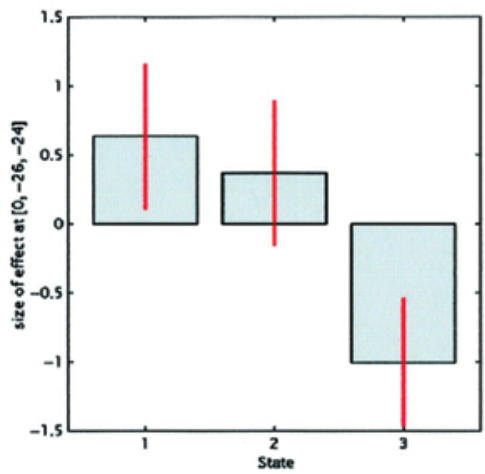

C. Pulvinar

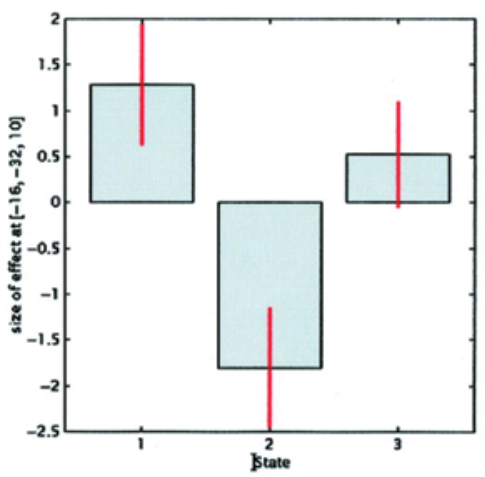

\section{B. Anterior cingulate cortex}

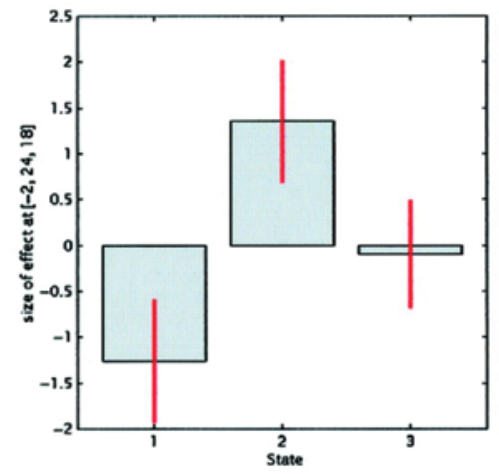

D. Cuneus

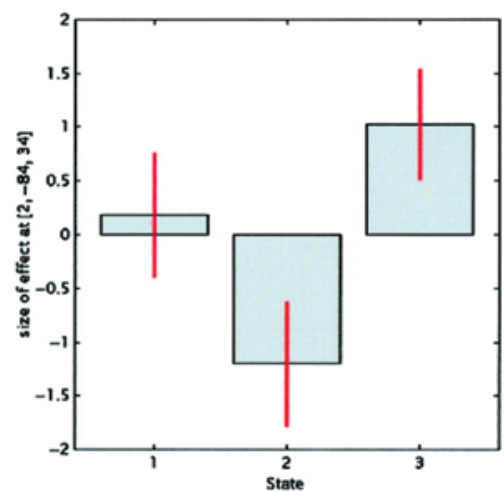

Fig. 6. Parameter estimates (or average relative changes in $\mathrm{rCBF}$ ) plotted against scanning conditions for the regions of interest: (A) dorsal rostral pons $(x=0, y= \pm 26, z= \pm 24)$; (B) anterior cingulate cortex $(x= \pm 2, y=24, z=18)$; (C) pulvinar ( $x$ $= \pm 16, y= \pm 32, z=10)$; (D) cuneus $(x=2, y= \pm 84, z=34)$. The scanning states were (1) stimulator at optimum settings: patient pain-free or with mild pain and paraesthesia; (2) stimulator off: patient in pain and no paraesthesia; (3) stimulator partially activated: patient with intermediate levels of pain and paraesthesia. Reproduced with permission of Goadsby \& Oxford University Press (16).

placement may fail, because cluster may switch sides (26). In a report of 2 cases, Schwedt et al (25) noted that despite dramatic relief of both cluster headache and of hemicrania continua, patients had persistence of autonomic phenomena such as lacrimation and rhinorrhea.

In a long-term outcome review by Schwedt et al (27) of 15 patients with multiple types of headache including chronic migraine (8), cluster headache (3), hemicrania continua (2), and post-traumatic headache (2), there were significant improvements in MI-
DAS scores, headache frequency, and severity as well as Headache Impact Test 6 (HIT-6) and Beck Depression inventory scores. Patients were implanted with percutaneous cylindrical leads. However, $60 \%$ required revision surgery for lead migration at 2 years and $100 \%$ required revision by 3 years, but the intervention otherwise was found to be both efficacious and safe (27).

A number of other case series, case reports, and reviews have reported favorable outcomes with occipital nerve stimulation for occipital neuralgia and 
cervicogenic headache as summarized in Tables 5 and 7. Hammer and Doleys (28) reported a case of intractable occipital neuralgia with deafferentation pain that failed multiple interventional treatments including an attempt at cervical spinal cord stimulation and classical approach at occipital nerve stimulation. Success was achieved only after a modified approach was employed, placing the percutaneous lead underneath the scar of a previous occipital neurectomy and ganglionectomy. The patient had near total relief of pain, marked improvement on the McGill Pain Questionnaire scores, and her Oswestry Disability scale improved from $46 \%$ to $0 \%$. The analgesic effect of ONS was persistent at 9 months after implant (28). Rodrigo-Royo et al (29) reported on 4 cases of cervicogenic headache (one post-herpetic) that were implanted with percutaneous lead type occipital neurostimulators. All patients had protracted chronic persistent severe headaches. ONS resulted in relief of continuous pain, a decrease in the frequency and severity of episodic pain, and a decrease in the consumption or discontinuation of analgesics Slavin et al (30) reported on 14 consecutive patients with intractable occipital neuralgia treated with ONS. Ten patients had successful trials and proceeded to implantation of the ONS. All had good pain relief at 6 months after implant. The mean follow-up period was 22 months. One patient had the system explanted due to infection and another one had lead migration and surgical revision with recapture of the stimulation but developed tightness and neck spasms and ultimately had the system explanted. Another patient had the system explanted 21 months after implant due to complete relief of pain obviating the need for stimulation. The same authors reported their experience again in a review form that included, in addition to ONS, infraorbital and supraorbital nerve stimulation for the management of craniofacial neuralgias. Two of the patients had a combination of a periorbital and an occipital stimulator, 13 had ONS, 4 had supraorbital, and 3 had infraorbital nerve stimulators. Significant relief occurred in the majority of patients (16 of 22), partial relief in 3 patients, and 3 patients continued to experience pain after device explantation for loss of effect or infection (31). Other case series have reported favorable experience with occipital stimulation in occipital neuralgia (32-34).

At the time of this writing, 2 prospective studies are pending on the use of occipital application of peripheral neurostimulation.

\section{Safety}

Lead displacement occurred frequently with percutaneous cylindrical leads, occurring in 30/115 patients reported and in 2 out of 35 patient with paddle type leads (Table 8). Battery failure due to depletion and requiring IPG replacement occurred often in the reported studies. The recent advent of rechargeable internal programmable generators has obviated that fact in clinical practice. There were 7 cases of infection reported in 150 implants (Table 8). Increased pain occurred in some patients at the lead or generator sites and patients occasionally requested explantation of the device. In 2 patients, total relief of pain and symptoms even with the device turned off, triggered the removal of the device. Gofeld (35) provided a technical report on an improved anchoring technique adding a distal anchor to hopefully reduce cylindrical lead migration.

\section{Rating the Overall Strength of the Body of Evidence}

The body of literature is considered taking in 4 prospective studies and multiple other reports. The results were consistently positive among the studies; there were no discrepancies to resolve. The magnitude of treatment results was overwhelmingly positive. While not included in the rating, 1 large retrospective narrative provides further support for the rating (5). The existing evidence for occipital application of peripheral nerve stimulation thus presents at least limited evidence, Level IV of V.

\section{Discussion}

The first reference in the literature for peripheral neurostimulation appears to be by Picaza et al in 1977 (15). However, the technique was popularized in the US after the case series report by Weiner and Reed in 1999 for treatment of occipital neuralgia with percutaneous leads (36). Despite the detailed description of what characterizes occipital neuralgia, the patients studied had origins of pain attributed to a variety of factors including closed head injury, auto accidents, cervical stenosis, cervical spondylosis, temporomandibular joint syndrome, ear infection, and idiopathic causes. The use of implanted neurostimulators in 13 patients in that report was based upon experience with other peripheral nerve applications of the technology and using headache classification by Ziegler and Murrow (37). Percutaneous leads were placed transverse to the para-sagittal course of the occipital nerves at 
the level of the arch of C1. Trial stimulation led to implantation in $100 \%$ of patients. All patients reported good to excellent relief in long-term follow-up. One patient underwent explantation following evidence of complete resolution of symptoms. Weiner (5) has subsequently published retrospective follow-ups of up to 150 patients claiming $70-75 \%$ long term success.

In this systematic review, there was one RCT examining the effects of occipital stimulation on PET scan patterns in the brain in 8 patients who were previously implanted with ONS and had successful outcomes. It placed 8 patients through 3 conditions of 1 variable, 4 times. There were also 9 observational studies, of which 4 were prospective and had adequate AHRQ scores to use in analysis. One of the prospective studies had 25 patients with $100 \%$ positive results (11); another study had 20 patients with $95 \%$ of patient having positive results (19). Other studies had smaller numbers of patients. Given the overwhelmingly and consistently positive results and uniformity of such positive findings in $70-100 \%$ of patients, the totality of the studies achieved meaningful results. The total number of patients in these prospective studies equals 83. There were several observational case series with pre- and post-treatment measurements of pain and function with modest numbers of patients. The positive outcome of included articles is echoed in 1 retrospective article by Weiner whereby he narrated his experience with 150 patients over a decade with positive results in over $70 \%$ of patients (5). The total number of patients reviewed in all of the articles considered, both included and excluded, is well over 300 with no negative studies.

It is difficult to construct an affordable and ethical double-blinded randomized controlled trial with sham versus effective stimulation. Patients implanted with spinal cord or peripheral nerve stimulators always perceive the paresthesia of stimulation, whereas patients subjected to sham procedures will not. The ethics of performing sham surgeries is considered improper by many. This leaves the possibilities of single cohort prospective studies or randomization to 2 non-blinded treatment options. The individual patient could serve as her/his own control; this has been repeatedly demonstrated when the stimulator is turned off or become dysfunctional as in the case of lead displacement or battery depletion. Loss of stimulation pattern or turning the stimulator off does result in loss of pain relief; thus efficacy of stimulation is at least empirically evident.

An alternative would be to implant both periph- eral nerve leads and high cervical spinal cord leads. Double-blinded controlled study could then be performed by switching stimulation from spinal cervical to peripheral lead stimulation. However, this approach compares 2 different stimulation techniques (high cervical spinal cord stimulation vs. ONS) but does not offer a true sham control to address the effectiveness of neurostimulation.

One could add to the strength of evidence with larger prospective observational studies and with the use of functional outcomes similar to those obtained in some of the ONS studies discussed in this review $(11,19,20,22)$. It would also be helpful to include annualized cost of treatment prior to implant and after implant to account for costs of medications, doctor visits, ER and hospital visits, missed work hours, and lost productivity. This latter data could be compared to a cohort of medically managed patients. The annualized direct medical expenses for chronic headache patients are likely to easily exceed the cost for appropriate use of peripheral neurostimulation, though this proposition has yet to be demonstrated. The gain for employers is potentially over tenfold the medical savings (2). Such data, proving cost effectiveness of spinal cord stimulation over re-operation or standard medical therapy has been garnered from studies of chronic pain patients with failed back surgery syndrome $(18,38)$, complex regional pain syndrome (39), and refractory angina (40).

ONS studies raise interesting inferences:

- $\quad$ Paddle lead use from the outset may be more cost effective due to lower need for lead revision due to displacement $(19,22)$.

- On the table trial may be cost effective compared to 2 trips to the operating room particularly since some series report $100 \%$ success with trials. Across the literature, the overall response to trial ranged from $71.5 \%$ to $100 \%(11,30)$.

- Regardless of true occipital neuralgia, cervicogenic headache, or transformed migraine, occipital nerve stimulation appears to work well for the relief of headache. Cluster headache and hemicrania continua also appear to respond, albeit a lower number of patients have been studied with less dramatic results; relief occurred slowly over time and autonomic phenomena appeared to persist (23-26).

- While occipital nerve block is used by many as a screening criterion there is no evidence that negative responders to ONB will fail or succeed 
with occipital nerve stimulation; in 1 retrospective study response or lack of response to ONB does not appear to matter (41).

- TENS did not predict implanted ONS outcomes (15).

- When using percutaneous cylindrical leads one may improve lead stability by placing anchors both proximally and distally (35).

- Peripheral stimulation may work even after occipital nerve transection (28).

- Measurement with headache scores including Headache Disability Questionnaire or MIDAS should provide better assessment than mere pain indices. When included in studies, functional scores and medication reduction were improved.

Other applications of peripheral field nerve stimulation have been applied successfully in the management of headache. Stimulation of subcutaneous peripheral branches of the trigeminal nerve has been successfully applied in case series to treat facial neuropathic pain mostly in the setting of traumatic or post-herpetic neuralgias $(42,43)$. Supraorbital nerve stimulation has also been used in the management of cluster headache in 1 case report (44). A retrospective study that examined patients who received vagus nerve stimulation for intractable epilepsy reported significant pain relief in 3 of 4 patients who had concomitant migraine (45).

\section{Conclusion}

Given the consistently positive results found in the qualifying studies, implantation of an ONS for headache should be offered to patients with frequent, intense, and impairing chronic headaches who have failed reasonable conservative treatment and interventional pain medicine efforts, and have undergone psycho-behavioral evaluation with a favorable profile. Improvements in equipment technology and refinement of implant techniques may make this useful treatment modality even more appealing.

\section{Acknowledgements}

The authors would like to thank the editorial board of Pain Physician for their constructive criticism in improving the manuscript and assistance in preparing the final version of the manuscript.

\section{References}

1. Stovner LJ, Hagen K. Prevalence, burden, and cost of headache disorders. Curr Opin Neurol 2006; 93:281-285.

2. $\mathrm{Hu} X \mathrm{XH}$, Markson LE, Lipton RB, Stewart WF, Berger ML. Burden of migraine in the United States: disability and economic costs. Arch Intern Med 1999; 1598:813-818.

3. Mathers CD, Stein C, Ma Fat D, Inoue $M$, Tomijima N. Global Burden of Disease 2000: Version 2 methods and results. GPE Discussion Paper No. 50. 2002. Geneva, World Health Organization.

4. Classification and WHO ICD-10NA Codes. Cephalalgia 2004; 24(S1):16-22.

5. Weiner RL. Occipital neurostimulation (ONS) for treatment of intractable headache disorders. Pain Medicine 2006; 7: S137-S139.

6. Goadsby PJ, Knight YE, Hoskin KL. Stimulation of the greater occipital nerve increases metabolic activity in the trigeminal nucleus caudalis and cervical dorsal horn of the cat. Pain 1997; 73:2328.

7. Bogduk N. Cervicogenic headache: Anatomic basis and pathophysiologic mechanisms. Curr Pain Headache Rep 2001; 5:382-386.
8. Suzuki N. Migraine-update-current concepts of migraine pathogenesis. Rinsho Shinkeigaku 2005; 45:834-836.

9. Le DK, Akerman S, Holland PR, Lasalandra MP, Bergerot A, Classey JD, Knight YE, Goadsby PJ. Occipital afferent activation of second order neurons in the trigeminocervical complex in rat. Neurosci Lett 2006; 403:73-77.

10. Piovesan EJ, Kowacs PA, Tatsui CE, Lange MC, Ribas LC, Werneck LC. Referred pain after painful stimulation of the greater occipital nerve in humans: Evidence of convergence of cervical afferences on trigeminal nuclei. Cephalalgia 2001; 21:107-109.

11. Popeney CA, Alo KM. Peripheral neurostimulation for the treatment of chronic, disabling transformed migraine. Headache 2003; 43:369-375.

12. West S, King V, Carey TS, Lohr K. Systems to rate the strength of scientific evidence. Evid Rep Technol Assess (Summ) 2002; 47:1-11.

13. Manchikanti L, Boswell MV, Giordano J. Evidence-based interventional pain management: Principles, problems, potential, and applications. Pain Physician 2007; 10:329-356.
14. Shealy CN, Mortimer JT, Reswick JB. Electrical inhibition of pain by stimulation of the dorsal columns: Preliminary clinical report. Anesth Analg 1967; 46:489-491.

15. Picaza JA, Hunter SE, Cannon BW. Pain suppression by peripheral nerve stimulation. Chronic effects of implanted devices. Appl Neurophysiol 1977; 40:223234.

16. Kemler MA, de Vet HC, Barendse GA, van den Wildenberg FA, van KM. The effect of spinal cord stimulation in patients with chronic reflex sympathetic dystrophy: Two years' follow-up of the randomized controlled trial. Ann Neurol 2004; 55:13-18.

17. Kemler MA, de Vet HC, Barendse GA, van den Wildenberg FA, van KM. Spinal cord stimulation for chronic reflex sympathetic dystrophy-five-year followup. N Engl J Med 2006; 354:2394-2396.

18. Kumar K, Malik S, Demeria D. Treatment of chronic pain with spinal cord stimulation versus alternative therapies: Costeffectiveness analysis. Neurosurgery 2002; 51:106-115.

19. Oh MY, Ortega J, Bellotte JB, Whiting DM, Alo K. Peripheral nerve stimulation 
for the treatment of occipital neuralgia and transformed migraine using a $\mathrm{C}_{1}$ 2-3 subcutaneous paddle style electrode: A technical report. Neuromodulation 2004; 7:103-112.

20. Melvin EA, Jr., Jordan FR, Weiner RL, Primm D. Using peripheral stimulation to reduce the pain of $\mathrm{C}_{2}$-mediated occipital headaches: A preliminary report. Pain Physician 2007; 10:453-460.

21. Matharu MS, Bartsch T, Ward N, Frackowiak RS, Weiner R, Goadsby PJ. Central neuromodulation in chronic migraine patients with suboccipital stimulators: A PET study. Brain 2004; 127:220-230.

22. Kapural L, Mekhail N, Hayek SM, Stanton-Hicks M, Malak O. Occipital nerve electrical stimulation via the midline approach and subcutaneous surgical leads for treatment of severe occipital neuralgia: A pilot study. Anesth Analg 2005; 10:171-174, table.

23. Burns B, Watkins L, Goadsby PJ. Treatment of medically intractable cluster headache by occipital nerve stimulation: Long-term follow-up of eight patients. Lancet 2007; 369:1099-1106.

24. Dodick DW. Occipital nerve stimulation for chronic cluster headache: Article and case report. Advanced Studies in Medicine 2003; 3:S569-571.

25. Schwedt TJ, Dodick DW, Trentman TL, Zimmerman RS. Occipital nerve stimulation for chronic cluster headache and hemicrania continua: Pain relief and persistence of autonomic features. Cephalalgia 2006; 6:1025-1027.

26. Magis $D$, Allena $M$, Bolla $M$, Pasqua VD, Remacle JM, Schoenen J. Occipital nerve stimulation for drug-resistant chronic cluster headache: A prospective pilot study. Lancet Neurol 2007; 6:314-321.

27. Schwedt TJ, Dodick DW, Hentz J, Trentman TL, Zimmerman RS. Occipital nerve stimulation for chronic headachelong-term safety and efficacy. Cephalalgia 2007; 27:153-157.

28. Hammer M, Doleys DM. Perineuromal stimulation in the treatment of occipital neuralgia: A case study. Neuromodulation 2001; 4:47-51.

29. Rodrigo-Royo MD, Azcona JM, Quero J, Lorente MC, Acin P, Azcona J. Peripheral neurostimulation in the management of cervicogenic headache: Four case reports. Neuromodulation 2005; 8:241248.

30. Slavin KV, Nersesyan H, Wess C. Peripheral neurostimulation for treatment of intractable occipital neuralgia. Neurosurgery 2006; 58:112-119.

31. Slavin KV, Colpan ME, Munawar N, Wess C, Nersesyan H. Trigeminal and occipital peripheral nerve stimulation for craniofacial pain: A single-institution experience and review of the literature. Neurosurg Focus 2006; 21: E5.

32. Johnstone CSH, Sundaraj R. Occipital nerve stimulation for the treatment of occipital neuralgia-eight case studies. Neuromodulation 2006; 9:41-47.

33. Waisbrod H, Panhans C, Hansen D, Gerbershagen HU. Direct nerve stimulation for painful peripheral neuropathies. J Bone Joint Surg Br 1985; 67-B:470-472.

34. Weiner RL, Alo KM, Fuller ML. Peripheral neurostimulation to control intractable occipital neuralgia. 9th World Congress on Pain, 61. 8-23-1999.

35. Gofeld M. Anchoring of suboccipital lead: Case report and technical note. Pain Pract 2004; 4:307-309.

36. Weiner RL, Reed KL. Peripheral neurostimulation for control of intractable occipital neuralgia. Neuromodulation. 1999; 2:217-221.

37. Ziegler DK, Murrow RW. Headache. In: Joynt R, editor. Clinical Neurology. J B
Lippincott, Philadelphia, PA 1988; 135 .

38. North RB, Kidd D, Shipley J, Taylor RS. Spinal cord stimulation versus reoperation for failed back surgery syndrome: A cost effectiveness and cost utility analysis based on a randomized, controlled trial. Neurosurgery 2007; 61:361-368.

39. Mekhail NA, Aeschbach A, StantonHicks M. Cost benefit analysis of neurostimulation for chronic pain. Clin J Pain 2004; 20:462-468.

40. Andrell P, Ekre O, Eliasson T, Bloomstrand $C$, Börjesson $M$, Nilsson $M$, Mannheimer C. Cost-effectiveness of spinal cord stimulation versus coronary artery bypass grafting in patients with severe angina pectoris-longterm results from the ESBY study. Cardiology 2003; 99:20-24.

41. Schwedt TJ, Dodick DW, Trentman TL, Zimmerman RS. Response to occipital nerve block is not useful in predicting efficacy of occipital nerve stimulation. Cephalalgia 2007;27:271-274.

42. Johnson MD, Burchiel KJ. Peripheral stimulation for treatment of trigeminal postherpetic neuralgia and trigeminal posttraumatic neuropathic pain: A pilot study. Neurosurgery 2004; 55:135141.

43. Slavin KV, Wess C. Trigeminal branch stimulation for intractable neuropathic pain: Technical note. Neuromodulation 2005; 8:7-13.

44. Narouze SN, Kapural L. Supraorbital nerve electric stimulation for the treatment of intractable chronic cluster headache: A case report. Headache 2007; 47:1100-1102.

45. Hord ED, Evans MS, Mueed S, Adamolekun B, Naritoku DK. The effect of vagus nerve stimulation on migraines. J Pain 2003; 4:530-534. 